\title{
Design and Implementation of Pulse Width Modulation Driving System for Voice Coil Motor
}

\author{
Rilong Liu ${ }^{1} \&$ Renxin Liu $^{1}$ \\ ${ }^{1}$ School of Engineering, Jiangxi Agricultural University, Nanchang, China \\ Correspondence: Rilong Liu, School of Engineering, Jiangxi Agricultural University, 1101 Zhimin Ave., \\ Nanchang, 330045, China. Tel: 86-791-83813184. E-mail: ckjx312@aliyun.com
}

Received: July 4, 2016

Accepted: August 18, 2016

Online Published: September 12, 2016

doi:10.5539/mas.v10n10p269

URL: http://dx.doi.org/10.5539/mas.v10n10p269

The research is financed by the Reform Project in Teaching of Jiangxi Province (JXJG-14-3-15), and Jiangxi Province's Academic Degrees and Graduate Education Reform Program (JXYJG-2015-056).

\begin{abstract}
According to the performance requirements of the control system for voice coil motor, a pulse width modulation driving system is designed and implemented based on an integrated power device and a field programmable gate array. LMD18200 is used as the motor driver to design the unit of switch type power driving. With APA300 as the digital controller, the modular design of the digital controller realizes with the Verilog hardware describing language, so it has a good flexibility and portability. The experimental results show that the current loop can rapidly keep track of current changes under the working condition of the voice coil motor power driver. Therefore, the servo system of voice coil motor is compared with the practical requirement.
\end{abstract}

Keywords: power driving, voice coil motor, servo system, module

\section{Introduction}

There is the interference modulation system in the atmospheric sounder. In order to driving the moving mirror to complete the swing motion, it is necessary to reasonably design the corresponding servo system, and enable the moving mirror to work in uniform motion with high precision in the effective stroke. The voice coil motor is characterized by its simple structure, small size, high speed and fast response speed. When combined with the control system, the voice coil motor can realize the speed servo control with high precision.

The servo system adopts the implementation scheme of double closed-loop control realized by the current loop and the speed loop, so that we can improve the stability of the moving mirror's scanning mechanism. Since the current loop is the inner loop, the transfer function of its control object has to be transformed. In this way, we can improve the rapidity of the system, and realize fast response to the control input of the speed loop (Zmood \& Holmes, 2003; Li \& Wu, 2009; Lin \& Li, 2012).

There are two types of driving modes in the power driving of voice coil motor. The first mode is linear amplification driving, which is not only power-consuming but also low efficient. It is common for voice coil motors of small power, usually under the wattage, to adopt this driving mode. The second mode is switch type driving, which is characteristic of low-power-consuming and fast response. The pulse width modulation is a commonly used method in the second driving mode. The pulse width modulation power driver employs the switch characteristics of high power transistors to modulate DC power supply of fixed voltage values. With a preset frequency value, we can switch on and off. By controlling the length of time needed to switch on and off in the transistor, we can control the average voltage flowing through the motor, and thus control the motor speed.

The motor controller has witnessed the development from the analog controller to the digital controller. The programmable logic device FPGA is known for its high integration, fast working speed and flexible programming. Hence FPGA is widely used in the driving control for the servo system of voice coil motor. Compared with discrete devices, the use of FPGA can increase the working frequency, simplify the circuit structure, and reduce the size and power consumption as well.

This paper puts forward a design of pulse width modulation power driver circuit based on the integrated power 
driving device LMD18200. In this proposed design, FPGA chip APA300 is the digital controller of choice, and full digital control is realized for pulse width modulation power driver. Experiments are conducted to verify the performance of pulse width modulation power controller in the servo system of voice coil motor (Yeom, Park \& Jung, 2007; Ke, Zhou, Yao \& Tang, 2003).

\section{Unit of Switch Type Power Driving}

With the pulse width modulation driving mode, the speed range is wide, the switch frequency is high, and the current waveform is good. The adoption of $\mathrm{H}$ type bipolar pulse width modulation driving can guarantee that the armature current is always continuous in the voice coil motor. This current enables the motor to generate high-frequency vibration, and to weaken the interference of static friction torque, so as to enhance the low speed features of the servo system. As a result, we adopt $\mathrm{H}$ type bipolar pulse width modulation driving mode in the voice coil motor power driving (Wu, Jiao, Yan, Zhang, Yu \& Chen, 2014; Wang, Guo, Yu \& Zhang, 2010). Considering factors like simplification of the circuit design, the system reliability and the driving module size, we choose the integrated power driving device LMD18200, whose internal function block diagram is shown in Figure 1.

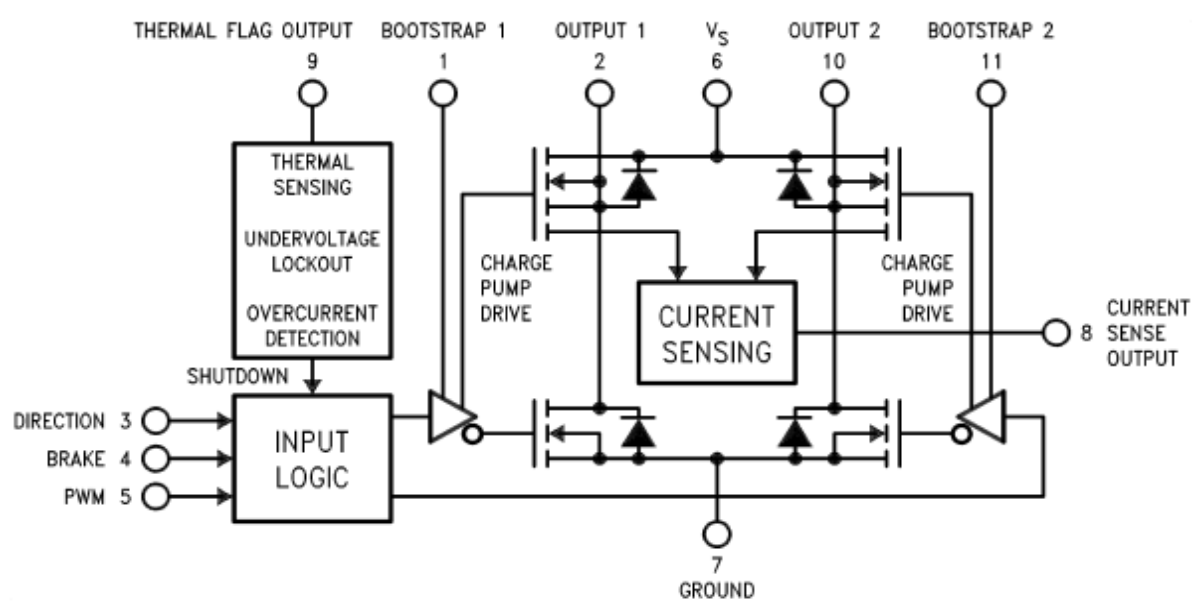

Figure 1. LMD18200's internal function block diagram

LMD18200, launched by National Semiconductor Corporation, is the H bridge assembly designed specifically for motion control. Integrated in a single chip are CMOS control circuit and DMOS power device. The peak output can run up to $6 \mathrm{~A}$, whereas the continuous output current can reach $3 \mathrm{~A}$. The operating voltage is able to reach up to $55 \mathrm{~V}$. Besides, there are also the temperature alarm, and overheating and short circuit protection functions (Puyal, Barragan, Acero, Burdio \& Millan, 2006). In the servo system for voice coil motor, the peak current is $1 \mathrm{~A}$. In the power driver, the operating voltage is $12 \mathrm{~V}$. Figure 2 below presents the power driving circuit composed by LMD18200.

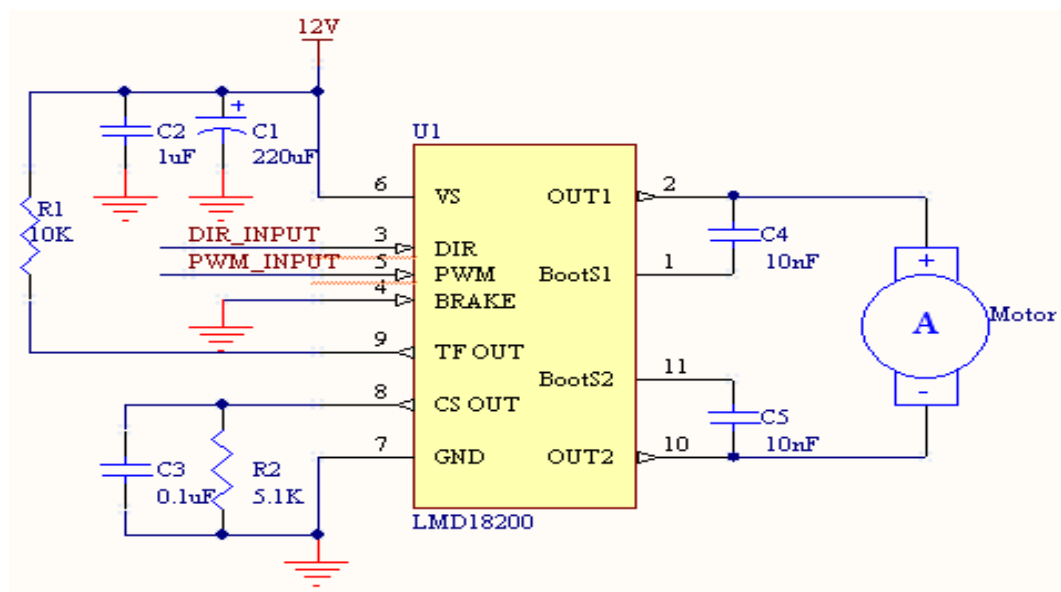

Figure 2. Power driving circuit 
Figure 1 shows that there are four DMOS power tubes inside LMD18200, making up a standard H driving bridge. When the internal charge pump circuit provides the switching tube of upper and lower bridge arm with gate control voltage, the switching frequency can reach as high as $500 \mathrm{KHz}$. Suppose the switching frequency for pulse width modulation is set at $25 \mathrm{KHz}$ in the servo system, we can use two $10 \mathrm{nF}$ bootstrap capacitors and charge these two bootstrap capacitor with DMOS tube. In this way, the switching tube is provided with gate control voltage.

The interference enters the motor controller in the form of spike pulse and noise. The FPGA output is digital PWM signal and DIR directional signal. The common mode interference is very likely to exist between these two types of signal and the analog signal. In order to prevent this from happening, photoelectric isolation technology is employed to suppress the interference in the servo system. Besides, wave of pulse width modulation and DIR directional signal serve as input to the power driving device LMD18200 after their being isolated by high speed optocoupler $6 \mathrm{~N} 137$. These steps can effectively separate the input and output channel from the controller, thus avoiding the formation of interference loops and preventing interference from entering into the internal system. In this way, the system can work reliably and stably.

\section{FPGA-based Digital Controller}

In the present servo system, the digital controller APA300 is the programmable Flash-based FPGA chip produced by Actel company. Compared with the SRAM-based FPGA chip, the built-in Flash unit has the secure and nonvolatile function of code storage. Therefore, no additional configuration chip is needed. When power is on, the chip is in operation (Monmasson, Idkhajine \& Naouar, 2011).

In FPGA-based digital controller, functions realized by FPGA can be divided into several independent modules in the servo system. Functions of each module are realized through programming of the Verilog HDL hardware description method. The modular design of the digital controller then comes into being. Figure 3 presents the schematics of digital controller based on FPGA.

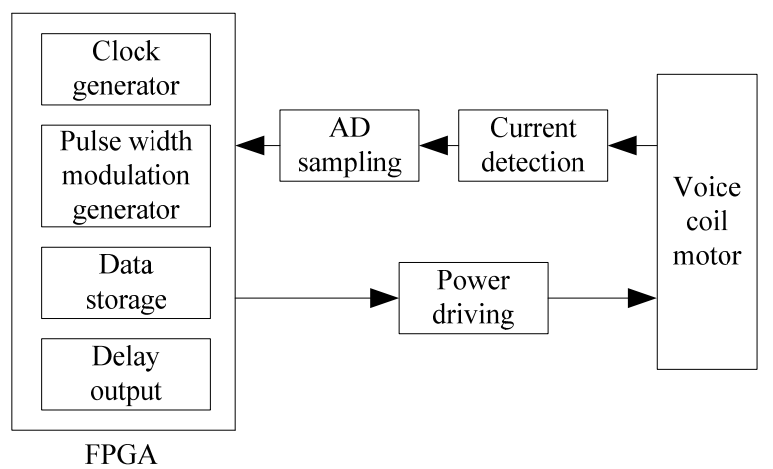

Figure 3. Schematics of FPGA-based digital controller

\subsection{Clock Generator Module}

The sequential logic circuit is composed of digital devices. This circuit's logic functions can be correctly utilized only under the effect of clock module. In the servo system for voice coil motor, two frequencies are needed: pulse width modulation switching frequency of the motor power driver and the directional control signal frequency.

Our system acquires the global system clock of $12.8 \mathrm{MHz}$ by calling phase locked loop kernel that comes with APA300. Frequency divider with different frequency coefficients is then designed by means of a counter. What we get is pulse width modulation switching frequency $25 \mathrm{kHz}$ and directional control signal $2 \mathrm{~Hz}$. According to application requirements, we can also change the count of the counter, so as to acquire different clock frequency signals and enhance the flexibility of the system application.

\subsection{Pulse Width Modulation Generator Module}

The speed servo control is achieved in voice coil motor when pulse width modulation power driving switching frequency remains invariant. It is through the current and speed negative feedback that its duty cycle is adjusted. Thus the tracking speed is realized. Ways to change the duty cycle include fixed frequency pulse width modulation method and fixed width frequency modulation method. 
Our system uses Verilog HDL programming and the method of fixed frequency pulse width modulation to change the duty cycle. Counters and comparators and registers are also employed to generate pulse width modulation wave. In other words, deviation between the actual value and the given value is compared with the fixed frequency's triangular wave generated by the add-subtract counter. After this comparison, pulse width modulation signal is successfully generated, which paves way for the acquisition of pulse width modulation wave characterized by its fixed switching frequency and adjustable duty cycle.

\subsection{Data Storage Module}

Static RAM is a commonly used data storage device. Compared with synchronous dynamic RAM, its reading and writing operations are convenient, and there is no need to refresh operation. Therefore, our system proposes a design based on static RAM chip CY7C1041 for data storage module.

Static RAM has only one set of data, address and control bus, which can be connected respectively to A/D converter and the digital controller through three-state buffer gate. When A/D converter takes sampling in the current, static RAM is linked to A/D converter by three-state gate, so that data are converted from A/D into static RAM. When the sampling is over, static RAM is switched by three-state gate to get connected to the digital controller, thus enabling the data to be read from static RAM. Additionally, SRAM can be accessed at random, and read and write at different times.

\subsection{Delay Output Module}

In order to ensure correct logic functions of the internal input in integrated power driving chip LMD18200, the input pulse width modulation signal and DIR directional signal should avoid edge alignment. In other terms, the edges of the two signals should have at least $1 \mu \mathrm{s}$ delay interval.

The implementation scheme is that FPGA hardware programming makes pulse width modulation signal delay $1 / 16$ pulse width modulation output cycle, so that wave of pulse width modulation and DIR signal have a delay along the edges. This is how the logic requirement of LMD18200 input signal is satisfied.

\section{Experimental Results}

The digital controller FPGA receives the current signal from the current sensor feedback. We can combine this signal with closed-loop control algorithm of the servo system to calculate corresponding pulse width modulation digital control quantity. Next power driving circuit is chosen to driving the motor.

The moving mirror's scanning mechanism requires forward and backward bidirectional movement. This means that, in the uniform scanning phase of the moving mirror, what the voice coil motor needs to do is to overcome the friction only. At the moment, the motor is in small current state of forward and bidirectional movement. In this dynamic process, the motor armature current has to be prevented from exceeding the allowable value. To put it in another way, no overshoot is expected with sudden control, or the smaller the overshoot the better. Hence, highly precise control of the small current is called for.

When the scanning mechanism is in uniform scanning phase, we measure its current curves, two cycles of which are shown in Figure 4.

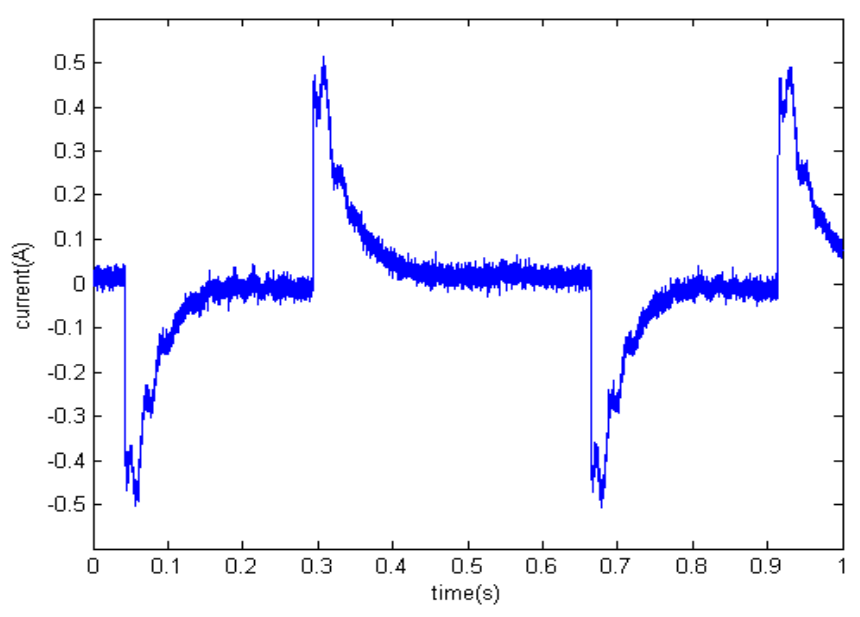

Figure 4. Current waveform of the voice coil motor 
It can be seen from Figure 4 that in uniform scanning, the current curve is smooth, and the current loop can rapidly keep track of current changes. All this reveals that pulse width modulation power driving in the servo system meet satisfyingly the system performance requirements.

\section{Conclusions}

By adopting the pulse width modulation driving mode for the voice coil motor power driving, this paper has designed a pulse width modulation driver based on the integrated power device LMD18200. With FPGA as the digital controller, we accomplish the task of full digital control of pulse width modulation power driver. Under the influence of pulse width modulation driver, the current loop can well track the system current, and improve the dynamic performance of the servo system for voice coil motor, thus satisfying the control requirements of the servo system.

\section{References}

Ke, J. W., Zhou, J. N., Yao, X. F., \& Tang, F. (2003). Design and Implementation of PWM Power Interface Used in Robot Motor Servo Control System. Journal of Kunming University of Science and Technology, 28(3), 85-89.

Li, W., \& Wu, Q. W. (2009). Design of Motion Control Platform Based on C8051F020 and LMD18200. Journal of Hubei University of Technology, 24(5), 78-80.

Lin, C. M., \& Li, H. Y. (2012). A Novel Adaptive Wavelet Fuzzy Cerebellar Model Articulation Control System Design for Voice Coil Motors. IEEE Transactions on Industrial Electronics, 59(4), pp. 2024-2033.

Monmasson, E., Idkhajine, L., \& Naouar, M. W. (2011). FPGA-based Controllers----Different Perspectives of Power Electronics and Drive Applications. IEEE Industrial Electronics Magazine, 5(1), 14-26. http://dx.doi.org/10.1109/MIE.2011.940250

Puyal, D., Barragan, L. A., Acero, J., Burdio, J. M., \& Millan, I. (2006). An FPGA-based Digital Modulator for Full- or Half-bridge Inverter Control. IEEE Transactions on Power Electronics, 21(5), 1479-1483. http://dx.doi.org/10.1109/TPEL.2006.880234

Wang, D. Y., Guo, H., Yu, K. P., \& Zhang, L. J. (2010). FPGA-based Power Drive Device for Voice Coil Motor Used in Direct Drive Valve. Journal of Beijing University of Aeronautics and Astronautics, 36(8), 953-956.

Wu, S., Jiao, Z. X., Yan, L., Zhang, R., Yu, J. T., \& Chen, C. Y. (2014). Development of a Direct-drive Servo Valve with High-frequency Voice Coil Motor and Advanced Digital Controller. IEEE Transactions on Mechatronics, 19(3), 932-942. http://dx.doi.org/10.1109/ / TMECH.2013.2264218

Yeom, D. H., Park, N. J., \& Jung, S. Y. (2007). Digital Controller of Novel Voice Coil Motor Actuator for Optical Image Stabilizer. IEEE International Conference on Control, Automation and Systems, pp. 2201-2206.

Zmood, D. N., \& Holmes, D. G. (2003). Stationary Frame Current Regulation of PWM Inverters with Zero Steady-state Error. IEEE Transactions on Power Electronics, 18(3), 814-822.

\section{Copyrights}

Copyright for this article is retained by the author(s), with first publication rights granted to the journal.

This is an open-access article distributed under the terms and conditions of the Creative Commons Attribution license (http://creativecommons.org/licenses/by/4.0/). 